\title{
Study on the potential effect of multi-AGC kinase AT13148 on the Notch signaling pathway in glioblastoma
}

Yanan $\mathrm{Li}^{1}$, Guosheng Han' ${ }^{1}$, Weijie Min ${ }^{1}$, Mengmeng Li ${ }^{2}$, Maomao Wang ${ }^{1}$, Chao Chen ${ }^{1}$, Yi Chen ${ }^{1}$, Laixing Wang ${ }^{1}$, Zhijian Yue ${ }^{1}$

\author{
'Department of Neurosurgery, Changhai Hospital, Second Military Medical University, \\ Shanghai, China \\ 2Department of Rheumatology, Changzheng Hospital, Navy Medical University, \\ Shanghai, China
}

Submitted: 13 November 2020; Accepted: 28 March 2021

Online publication: 4 April 2021

Arch Med Sci

DOI: https://doi.org/10.5114/aoms/135083

Copyright @ 2021 Termedia \& Banach

\section{Abstract}

Introduction: Glioblastoma is the most malignant astrocytoma, and the efficacy of its therapy is not ideal. The Notch signaling pathway plays an important role in tumor proliferation and invasion. Whether the small molecule drug AT13148 can affect glioblastoma by regulating the Notch signaling pathway is the focus of this study.

Material and methods: In vitro, the glioblastoma U87 cell line transfected with sh-ITGB1 (U87 $7^{\text {sh-ITGB1}}$ ), the U87 cell line transfected with oe-ITGB1 (U87 $7^{\mathrm{Oe}-\mathrm{TGB} 1}$ ) and the control group were treated with the small molecular drug AT13148. RT-qPCR, western blot and clone formation ability assays were used to detect the mRNA and protein expression of the ITGB1 and the key gene NOTCH1, as well as the proliferation of cancer cells. Therapeutic effects of AT13148 were examined in vivo using a nude mouse model of U87 cells. After treatment with AT13148, volume of tumors was calculated, and RT-qPCR and western blot were used to evaluate the mRNA and protein expression of ITGB1 and NOTCH1.

Results: AT13148 inhibits the activity of U87 cells. Lentiviral transfection of sh-ITGB1 and oe-ITGB1 can interfere with the expression of ITGB1 in U87 cells. AT13148 could down-regulate both the expression of ITGB1 and NOTCH1. Moreover, AT13148 affects the cloning ability of U87 cells. AT13148 can also inhibit the proliferation of U87 cells. Furthermore, AT13148 inhibited the proliferation and invasion of transplanted tumors in vivo.

Conclusions: This study indicated that AT13148 could affect the expression of ITGB1 and NOTCH1, which also could be a potential anti-glioblastoma small molecule drug candidate in clinical medicine.

Key words: glioblastoma, small molecule drug, AT13148, Notch, ITGB1, NOTCH1.

\section{Introduction}

Gliomas originate from neuroepithelial tissues, most of which are malignant [1]. Gliomas include astrocytoma, oligodendroglioma, ependymoma, mixed gliomas, and neuroepithelial tumors [2]. According to the malignant degree of the tumors, the WHO classifies gliomas from grade I (low proliferative capacity, low invasive astrocytoma and oligodendro-

\author{
Corresponding authors: \\ Zhijian Yue \\ Department of Neurosurgery \\ Changhai Hospital \\ Second Military Medical \\ University \\ No. 168 Changhai Road \\ Shanghai 200433, China \\ Phone/fax: \\ +86-021-31161765 \\ E-mail: ypv8droe@sina.com \\ Laixing Wang \\ Department of Neurosurgery \\ Changhai Hospital \\ Second Military Medical \\ University \\ No. 168 Changhai Road \\ Shanghai 200433, China \\ Phone/fax: \\ +86-021-31161765 \\ E-mail:wlx920@163.com
}


glioma) to grade IV (highly invasive, mitotic active, necrotizing glioblastoma) [3]. For malignant glio$\mathrm{ma}$, the preferred treatment is still surgical treatment, which can achieve the total removal of tumors within a controlled range [4]. When tumors invade functional areas or conduction tracts, they need to be operated on with the help of neuronavigation, functional magnetic resonance imaging, or even wake-up during the operation for functional protection [5]. Regular radiotherapy and chemotherapy are administered after the operation. At present, the most commonly used chemotherapeutic drug is temozolomide. The dosage of temozolomide is related to the patient's body surface area, and there are differences between the period of radiotherapy and after radiotherapy [6]. Although many new techniques have been developed to improve the resection of glioblastoma, the bioavailability and blood brain concentration of temozolomide can reach a good state after the operation, but for the overall prognosis of glioblastoma, there is no significant improvement. The use of chemoradiation, immunotherapy, and radio sensitizers as adjuvant therapy cannot reduce the high rates of recurrence within a few months after treatment $[7,8]$. After glioblastoma diagnosis, in patients without treatment overall survival is typically 3 months. A study reported that the 10-year survival rate of glioblastoma was only $0.71 \%$ [9]. In addition to actively improving surgical techniques, scientists have also explored new treatment methods from various aspects, such as small molecule drugs, immunotherapy, photodynamic therapy and so on, but the effect is not satisfactory [10-12].

The Notch signaling pathway occurs widely in vertebrates and invertebrates and is a highly conserved signaling pathway. It plays a crucial role in cell proliferation and apoptosis [13]. Astragaloside IV (AS-IV) effectively reversed hypoxia-induced pulmonary vascular remodeling and pulmonary artery smooth muscle cell proliferation via the Notch signaling pathway [14]. It has been reported that Notch signaling pathway can not only regulate cell proliferation and differentiation, but also interact with other signaling pathways and growth factors [15]. The crosstalk between Notch and mitogen-activated protein kinase (MAPK) pathway plays a role in MEK inhibitor resistance in BRAFV600E metastatic melanoma and promotes migration in GNAQQ209L uveal melanoma (UM) cells [16]. A study confirmed that miR-223 can inhibit the expression of F-box and WD repeat domain-containing7 (FBXW7), and ultimately increase the proliferation and apoptosis of colorectal cancer cells through Notch and Atk/mTOR signaling pathways [17]. The abnormal expression of the Notch signaling pathway can lead to vascular malformation in mice [18]. The Notch signaling pathway has become a very important target in cancer therapy. It has been found that activation of the Notch signaling pathway is associated with the development of glioblastoma [19]. The proliferation and cloning ability of glioma stem cells can be significantly enhanced by over-expression of Notch1 intracellular domain (NICD) [20]. The most influential gene integrin $\beta 1$ (ITGB1) was previously identified by screening for genes that changed after the Notch signaling pathway was activated in glioma [21]. ITGB1 is a member of the integrin family, which is formed by $18 \alpha$ and $8 \beta$ transmembrane subunits. ITGB 1 is a transmembrane receptor that mediates the connection between cells and their external environment [22]. It can activate the PI3K/Akt pathway and p130cas/ JNK signal transduction pathway, and regulate cell proliferation, invasion and migration [23].

AT13148 is a new oral competitive multi-target kinase inhibitor of adenosine triphosphate (ATP). It was found that AT13148 affected the phosphorylation of rho-associated kinase (ROCK), protein kinase $B(P K B)$, p70S6K and protein kinase (PKA) substrates, thus inducing apoptosis of cancer cells [24]. Inhibition of recombinant glutamate cysteine ligase modifier (GCLM) by AT13148 can improve the prognosis of chemotherapy in glioma patients. In previous research, we found that AT13148 was closely associated with seven genes in the Notch signaling pathway, including ITGB1 [21]. A previous study showed that Mega-Itgb1 modulated angiogenesis upstream of Notch signaling [25]. Whether AT13148 can inhibit ITGB1 and further regulate the Notch signaling pathway, thus affecting the proliferation and invasion of glioblastoma, needs more research. In this study, we aimed to explore the regulatory action of AT13148 on ITGB1 and its effect on glioblastoma and provide some ideas for clinical treatment of glioblastoma.

\section{Material and methods}

\section{Cell line and culture}

U87 human glioma cells were obtained from the First Affiliated Hospital of the Naval Military Medical University (Shanghai, China) and routinely cultured in DMEM (Gibco, US) included with $10 \%$ fetal calf serum (Gibco, USA) and at $37^{\circ} \mathrm{C}$ in an incubator (Jingsheng Instrument, China) with a humidified atmosphere and $5 \% \mathrm{CO}_{2}$.

The culture medium was removed and PBS was used to wash the cells for subculture. After adding $0.25 \%$ trypsin, the cells were placed in an incubator (Jingsheng Instrument, China) at $25^{\circ} \mathrm{C}$ for $1 \mathrm{~min}$. When there were free-floating cells, the cell suspension was moved to a centrifugal tube (AxyGen PCR-02-C, USA), and was centrifuged for 
3 min at 500 rpm (Eppendorf 5417R, Germany). Samples with fresh culture medium were resuspended and counted, and then inoculated in a petri dish.

\section{Optimum concentration of AT13148 assay}

The $U 87$ cell suspension (10 000 cells/100 $\mu \mathrm{l} /$ well) was inoculated into a 96-well plate. The culture plate was placed in an incubator (Thermo Forma, USA) for $24 \mathrm{~h}\left(37^{\circ} \mathrm{C}, 5 \%\left(\mathrm{CO}_{2}\right)\right.$. Different concentrations of AT13148 $(50 \mu \mathrm{M}, 20 \mu \mathrm{M}, 10 \mu \mathrm{M}$, $5 \mu \mathrm{M}, 2 \mu \mathrm{M}, 1 \mu \mathrm{M}, 500 \mathrm{nM}, 300 \mathrm{nM}, 50 \mathrm{nM}$, $20 \mathrm{nM}, 10 \mathrm{nM}$, control, Selleck, China) were put in the 96-well plate with the U87 cell suspension for $24 \mathrm{~h}$. Then cells were incubated with $10 \mu \mathrm{l}$ of CCK-8 (Yeasen, China) in an incubator (Jingsheng Instrument, China) for $4 \mathrm{~h}$. Lastly, the absorbance value (OD) at $450 \mathrm{~nm}$ wavelength was measured by an enzyme-labeled instrument (Bio-tek, USA). Cell viability was quantified with the following formula: (ODexp - ODblank)/(OD $\left.{ }_{n c}-O_{\text {blank }}\right) \times 100 \%$. Each sample was measured three times and each sample had three biological replicates. AT13148 (water insoluble, 313.78, $\mathrm{C}_{17} \mathrm{H}_{16} \mathrm{CIN}_{3} \mathrm{O}$ ) was purchased from MedChemExpress (USA) with purity of $99.54 \%$.

\section{Lentivirus packaging and transfection}

The DNA-Lipofectamine 2000 complex was prepared according to the Lipofectamine 2000 protocol [26]. The medium of U87 cell suspension was removed, and we added $2 \mathrm{~mL}$ of lentivirus medium with DMEM and $10 \%$ thermally inactivated fetal bovine serum (HI-BS) for $48 \mathrm{~h}$. After that operation, the U87 cells were transferred to medium with DMEM, $10 \% \mathrm{HI}$-FBS, $1 \%$ penicillin/streptomycin (P/S) and $0.5 \mathrm{mg} / \mathrm{Ml}$. U87 was cultured in the medium without antibiotics until the density reached $30 \%$. The $293 \mathrm{~T}$ cells were transfected by an overexpression plasmid (oe-ITGB1) and an interference expression plasmid (sh-ITGB1). Inclusion of the green fluorescent protein (GFP) gene in the vector smooths the tracking of any transduction in subsequent experiments (Supplementary Figure S1). Viruses made from 293T cell were collected, and filtered by $0.5 \mu \mathrm{m}$ microporous membrane; $1 \mathrm{ml}$ of lentivirus medium and $1 \mathrm{ml}$ of virus solution were added to U87 cells in the presence of $4 \mu \mathrm{g} / \mathrm{ml}$ polybrene. Then the cells were transferred to the objective cell culture me- dium containing DMEM and 10\% FBS without antibiotic. The expression of GFP was detected by fluorescence microscope (CKX41; Olympus, Tokyo, Japan) after transfection of oe-ITGB1 empty vector, oe-ITGB1 vector, sh-ITGB1 empty vector and sh-ITGB1 vector for 72 hours in order to determine the transfection efficiency.

\section{RNA extraction and $q R T-P C R$}

RNA was isolated from cells or tumor tissues using Trizol (Invitrogen, USA) following the manufacturer's protocol. The isolated RNA was transcribed into cDNA using the PrimeScript RT Reagent Kit with gDNA Eraser (Takara, Japan). The $20 \mu \mathrm{l}$ reaction system consisted of $10 \mu$ l of genomic DNA removal solution, $1 \mu$ l of PrimeScript RT Enzyme Mix I, $1 \mu$ l of RT Primer Mix, $4 \mu$ of $5 \times$ PrimeScript Buffer 2 , and $4 \mu \mathrm{l}$ of RNase Free $\mathrm{dH}_{2} \mathrm{O}$. The reverse transcription conditions performed on the Eppendorf Mastercycler X50a (Eppendorf, Germany) were as follows: $37^{\circ} \mathrm{C}$ for $15 \mathrm{~min}, 85^{\circ} \mathrm{C}$ for $5 \mathrm{~s}$, and $4^{\circ} \mathrm{C}$ for $15 \mathrm{~min}$. All primers used in this study are listed in Table I. In this study, all the primers were synthesized by Sangon Biotech, Shanghai, China. qRTPCR was performed using SYBR Primer Ex Taq $\Pi$ (Takara, Japan) on a ViiA 7 system (Applied Biosystems, USA) and the operation process was carried out according to the manufacturer's protocol. The $20 \mu \mathrm{l}$ reaction system consisted of $10 \mu \mathrm{l}$ of SYBR Premix Ex Taq, $0.4 \mu$ of ROX Reference Dye II, $0.8 \mu \mathrm{l}$ of forward primer and reverse primer, $4 \mu \mathrm{l}$ of cDNA, and $4 \mu \mathrm{l}$ of $\mathrm{ddH}_{2} \mathrm{O}$.

The reaction consisted of 40 cycles and the procedure was as follows: $95^{\circ} \mathrm{C}$ for $30 \mathrm{~s}, 95^{\circ} \mathrm{C}$ for $5 \mathrm{~min}, 60^{\circ} \mathrm{C}$ for $34 \mathrm{~s}, 95^{\circ} \mathrm{C}$ for $15 \mathrm{~s}, 60^{\circ} \mathrm{C}$ for $1 \mathrm{~min}$, and $95^{\circ} \mathrm{C}$ for $15 \mathrm{~s}$. Each group consisted of three biological replicates with three technical replicates per sample. GAPDH was used for normalizing different samples as an internal control. The calculation formula is as follows: $\Delta \Delta \mathrm{CT}=\Delta \mathrm{CT}$ (target gene) $-\Delta C T$ (reference gene).

\section{Western blot assay}

Cell lysates were prepared using RIPA buffer (Santa Cruz Biotechnology). After centrifugation at $12000 \mathrm{rpm}$ at $4^{\circ} \mathrm{C}$ for $20 \mathrm{~min}$, supernatants were collected and protein concentrations were determined by the DC Protein Assay Kit (Bio-Rad Laboratories). Cell protein lysates were separated for 70 min by $15 \%, 10 \%$ and 5\% SDS-PAGE with

Table I. Primer sequences for qRT-PCR

\begin{tabular}{|lcc|}
\hline Gene & Forward $\left(\mathbf{5}^{\prime} \mathbf{-} \mathbf{3}^{\prime} \mathbf{)}\right.$ & Reverse $\mathbf{( 5}(\mathbf{v e r}))$ \\
\hline ITGB1 & CCTTGGGATGACTTGATTG & CCTTTGCTACGGTTGGTTACAT \\
\hline NOTCH1 & GAGGCGTGGCAGACTATGC & CTTGTACTCCGTCAGCGTGA \\
\hline GAPDH & GGAGCGAGATCCCTCCAAAAT & GGCTGTTGTCATACTTCTCATG \\
\hline
\end{tabular}


$80 \mathrm{~V}$, and then transferred onto the polyvinylidene fluoride (PVDF) membranes (Immobilon; Millipore, Bedford, MA, USA) using a semidry system for $1 \mathrm{~h}$ at $300 \mathrm{~mA}$. Polyvinylidene difluoride membranes were blocked with 5\% skim milk (Wako Pure Chemical Industries, Osaka, Japan) in phosphate buffer saline (PBS) at $25^{\circ} \mathrm{C}$ for $1 \mathrm{~h}$. Then they were incubated with the primary antibodies, diluted in $5 \%$ skim milk at $37^{\circ} \mathrm{C}$ for $2 \mathrm{~h}$. The membrane was washed and incubated with the following primary antibodies: NOTCH 1 (Affinity, AF5307, 1:500), ITGB1 (Affinity, BF0036, 1:500), GAPDH (Abcam, ab8245, 1:1000). Secondary antibodies used were Goat anti Rabbit IgG $(\mathrm{H}+\mathrm{L})$ HRP (S0001, 1:10000) and Goat anti-Mouse IgG $(\mathrm{H}+\mathrm{L}) \mathrm{CY} 3$-conjugated (S0012, 1:100). After the washing operation, enhanced chemiluminescence assays (NEN Life Science Products, Boston, MA, USA) were performed, and all the positive bands were identified on Fuji Medical X-ray film.

\section{Colony formation assay}

The cells were suspended in complete growth medium with $0.3 \%$ low-melting agarose, then cells were transferred into solidified $0.6 \%$ agarose in six-well culture plates $\left(2 \times 10^{4}\right.$ cells). The medium was changed every three days. Two weeks after incubation, using the Omnicon 3600 image analysis system the numbers of colonies was counted. The colonies were stained using a $0.04 \%$ crystal violet solution for $2 \mathrm{~h}$ and visualized. Each group consisted of three biological replicates.

\section{Xenograft tumor in nude mice}

All the experiments performed in this study were approved by the Experimental Animal Ethics Committee at the Second Military Medical University (Shanghai, China). Pathogen-free, female, 4-week-old BALB/c-nu (nude) mice were purchased from Charles River Laboratories (Wilmington, MA, USA). To analyze the effect of AT13148 on tumor

A

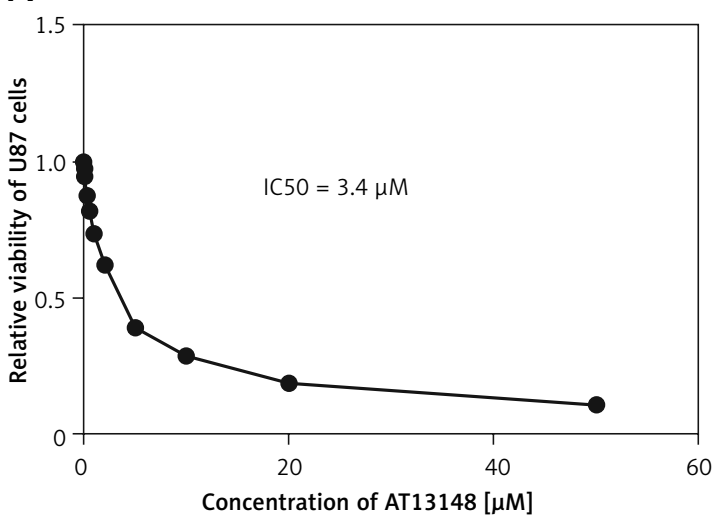

cells, U87 cells $\left(6 \times 10^{6}\right)$ were injected into the mice. After 3 weeks of feeding, the mice with tumors were randomly divided into a control group and an experimental group with 6 mice in each group. The mice in the experimental group were given $45 \mathrm{mg} / \mathrm{kg} /$ day AT13148, while the mice in the control group were given $45 \mathrm{mg} / \mathrm{kg} /$ day physiological saline. After 12 days, animals were sacrificed as per institutional guidelines. Body weight and tumor volume were measured over the whole time of the experiment every 3 days. Volumes of the tumor were measured with the equation: volume $=($ length $\times$ width $\times$ height/2) .

\section{Immunohistochemical assay}

For immunohistochemical staining, slides were created from paraffin-embedded murine specimens. Each group contained six slices. The slices were immersed in xylene for $15 \mathrm{~min}$, then dehydrated in ethanol and washed with PBS. Antigen repair was carried out according to the instructions of the primary antibody manufacturer. The primary antibodies of NOTCH 1 (Affinity, AF5307, 1:500) and ITGB1 (Affinity, BF0036, 1:500) were used to incubate slides, for $12 \mathrm{~h}$ at $4^{\circ} \mathrm{C}$ and $1 \mathrm{~h}$ at $25^{\circ} \mathrm{C}$. Following a washing step, all slides were incubated with secondary antibodies in blocking solution for $1 \mathrm{~h}$ at room temperature. After PBS was removed, DAB was added to the slices and incubated for $10 \mathrm{~min}$.

Distilled water was used to wash the slices, and then hematoxylin was used for re-staining. The slides were assessed under a microscope (BX51M, OLYMPUS, Tokyo, Japan).

\section{Statistical analysis}

All data in this study were analyzed by SPSS version 20.0. Two-tailed Student's $t$-test was used to do the significance analysis. Error bars represent the error of the mean ( \pm SD). For whole analyses, the value of $p<0.05$ was deemed as significant.

B

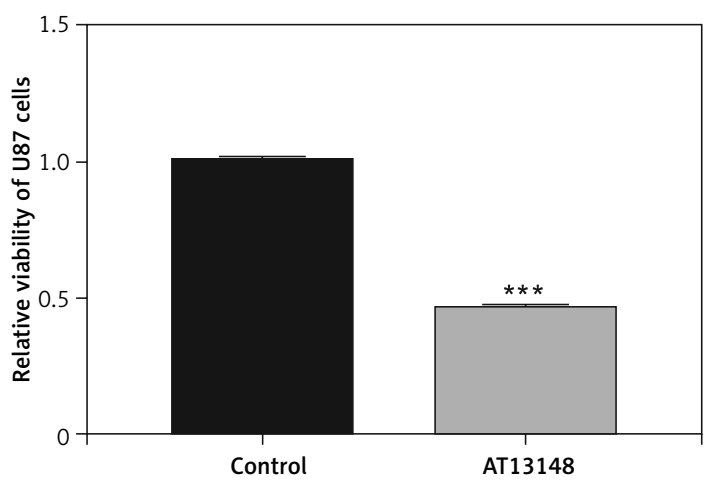

Figure 1. Relative viability of U87 cells. A - Relationship between AT13148 concentration and U87 cell viability. B - Relative viability of U87 cells in control group and AT13148 treatment group, ${ }^{* * *} p<0.001$ 


\section{Results}

AT13148 inhibits the activity of U87 cells

We used a CCK-8 kit to measure proliferation of U87 cells treated with different concentrations of AT13148. According to the concentration curve (Figure $1 \mathrm{~A}$ ), the $I C_{50}$ concentration of AT13148 was $3.4 \mu \mathrm{M}$. We treated U87 cells with $3.4 \mu \mathrm{M}$ AT13148, and found that compared with the control group, the relative viability of the AT13148 group was inhibited by $54 \%(p<0.001$, Figure $1 \mathrm{~B})$.

A

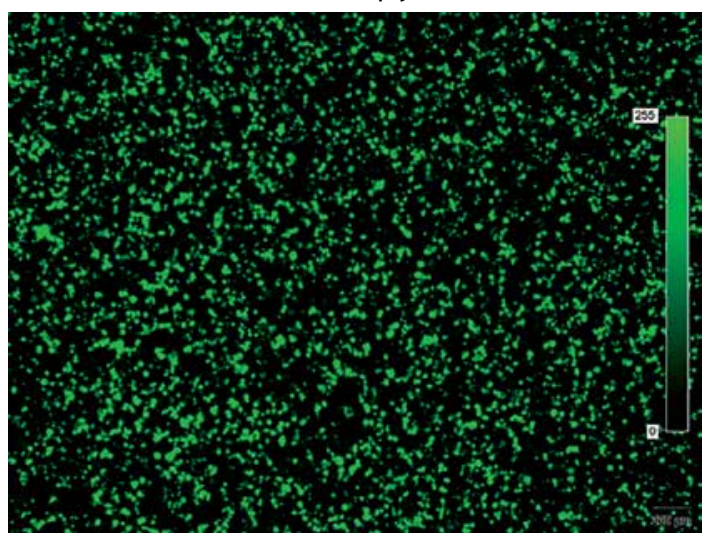

sh-ITGB1 empty vector

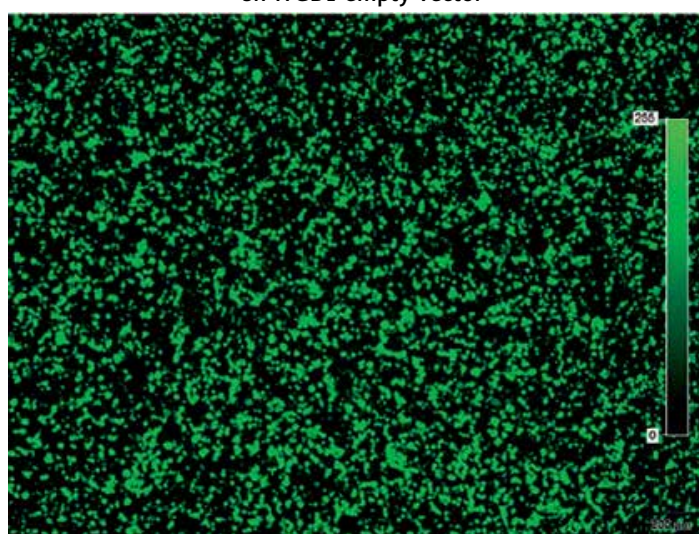

B

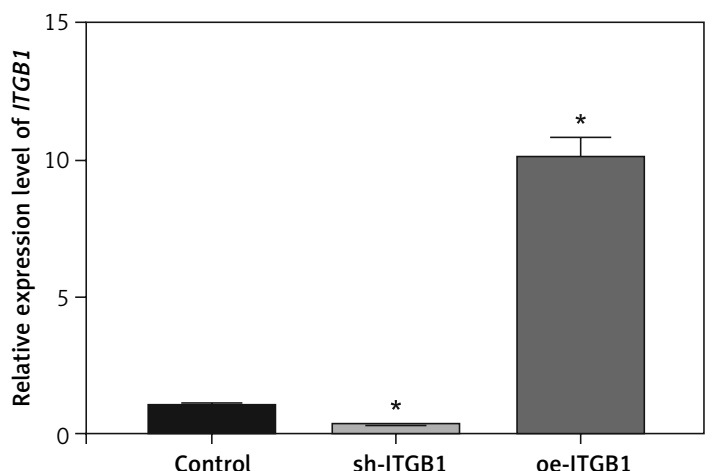

Lentivirus transfection of sh-ITGB1

and oe-ITGB1 can interfere with the expression of ITGB1 in the U87 cell line

Transfection efficiency was determined by fluorescence microscopy, and the result indicated that the transfection efficiency of sh-ITGB1 and oe-ITGB1 viruses was high, and the viruses could be used in the further experiments (Figure $2 \mathrm{~A}$ ). As shown in Figure $2 \mathrm{~B}$, the relative expression level of ITGB1 in the sh-ITGB1 group was significantly

oe-ITGB1

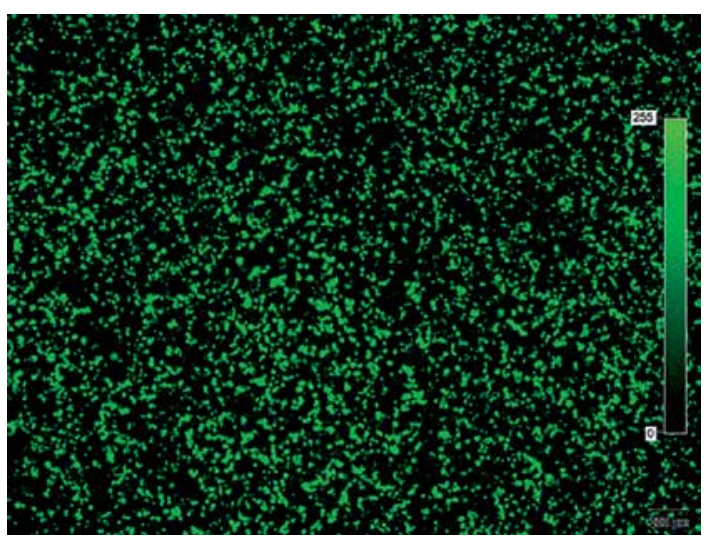

sh-ITGB1

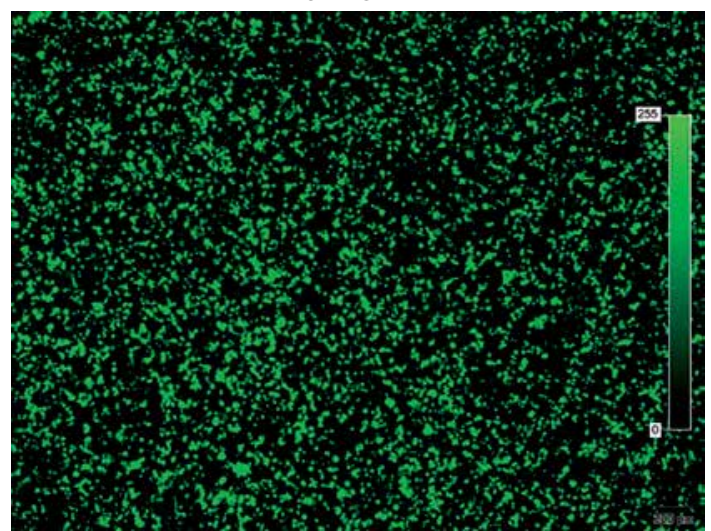

C

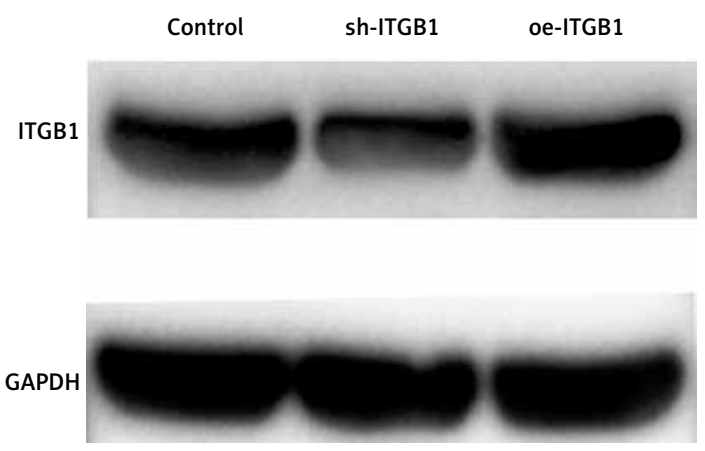

Figure 2. Transfection of U87 cells with lentivirus for overexpressed (oe-ITGB1) and interference expressed ITGB1 (sh-ITGB1). A - Transfection efficiency of lentivirus in the oe-ITGB1 with empty vector, oe-ITGB1, sh-ITGB1 with empty vector, and sh-ITGB1 was detected by fluorescence microscope. B - Relative expression level of ITGB1 in the control, sh-ITGB1, and oe-ITGB1 group. C - Relative protein expression level of ITGB1 in the control, sh-ITGB1, and oe-ITGB1 group 
lower than that in the control group $(p<0.05)$, while it was higher in the oe-ITGB1 group $(p<$ $0.05)$. After transfection of U87 cells with lentivirus, the expression of ITGB1 protein in the experimental group and control group changed (Figure 2 C). Therefore, lentiviral transfection of sh-ITGB1 and oe-ITGB1 can interfere with the expression of ITGB1 in U87 cells.

\section{AT13148 regulates both ITGB1 and NOTCH1}

U87, U87 $7^{\circ-1 T G B 1}$, and U87 $7^{\text {sh-ITGB1}}$ cells were treated with AT13148 with the concentration $I_{50}$. The relative expression level of ITGBI in U87 $7^{\text {oe-ITGB1}}$, U $87^{\text {sh-ITGB1 }}$ and the U87 group treated with AT13148 was significantly lower than that in the control group $(p<0.05)$ after $48 \mathrm{~h}$ culture (Figure $3 \mathrm{~A}$ ). The expression of the gene ITGB1 in U87 $7^{\text {sh-ITGB1 }}$ cells treated with AT13148 was the lowest. From the results of western blot, we can see that the protein expression of ITGB1 in each experimental group decreased to varying degrees compared with that in the control group (Figure 3 C). All the results showed that AT13148 had a regulatory effect on ITGB1.

The same research method was used to study the effect of AT13148 on NOTCH1. As shown in Figure 3 B, after treatment with AT13148, the expression of NOTCH1 was significantly decreased in the U87 8 h-ITGB1 cells and U87 cells compared

\section{A}

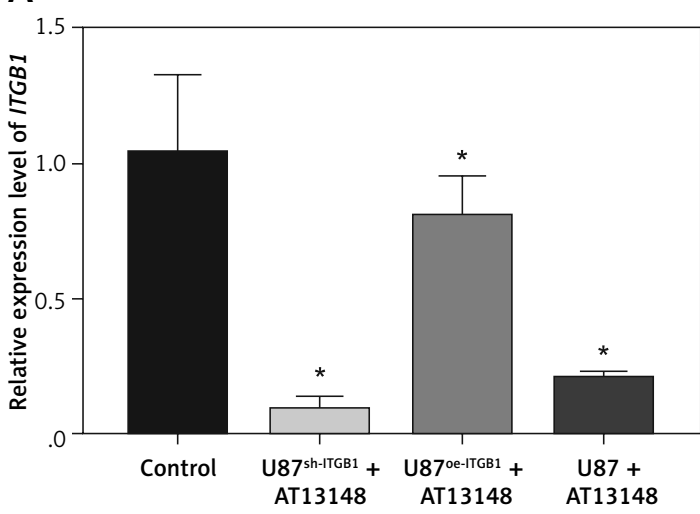

C ITGB1

GAPDH
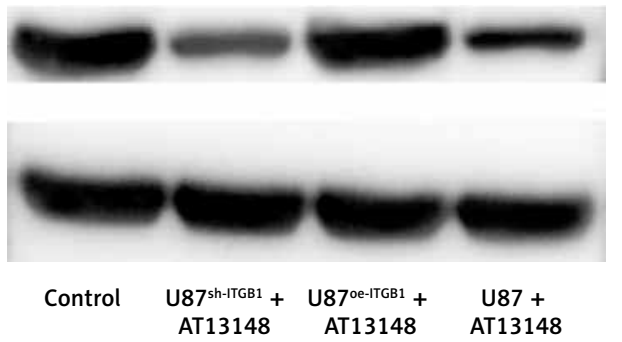

with that in the control group $(p<0.05)$. Figure $3 \mathrm{D}$ shows that AT 13148 also affected the protein expression of NOTCH1.

\section{AT13148 inhibits the clone formation ability of U87 cells}

To test whether AT13148 had an effect on the proliferation of glioblastoma cells, colony formation assay was carried out (Figure 4) in the U87 cells. After treatment with AT13148, the number of clones in the U87 group was significantly lower than that in the control group without AT13148 $(p<0.05)$. There was no significant difference in the number of clones between the U87 ${ }^{\circ-I T G B 1}$ after being treated with AT13148 and the control group $(p>0.05)$, while the number of clones in the group of U87 $7^{\text {sh-ITGB1 }}$ after being treated with AT13148 was significantly lower than that in the control group $(p<0.05)$.

\section{AT13148 affects the volume of tumors in nude mice}

In this study, the nude mouse model of glioblastoma U87 was established by subcutaneous injection. Twelve mice were successfully modeled (Figures 5 A, B). During AT13148 treatment, there was no significant difference in the body weight of nude mice between the experimental group and the control group $(p>0.05)$, and it ranged from

\section{B}

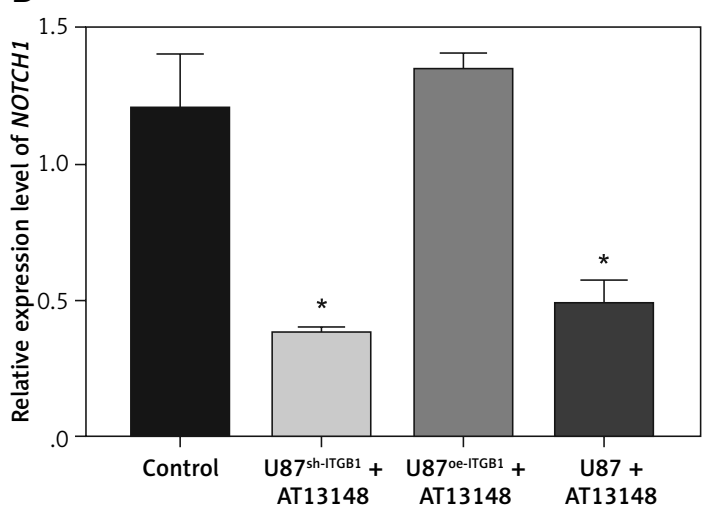

D

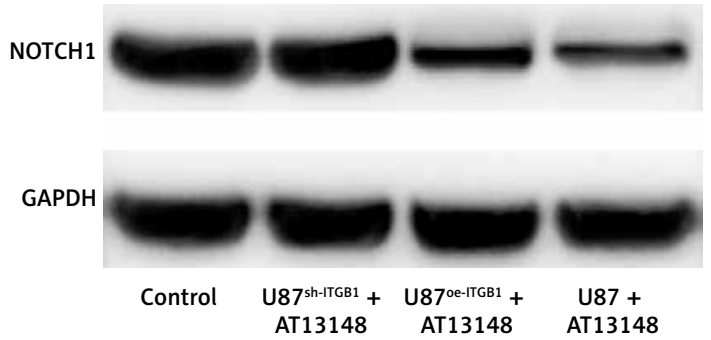

Figure 3. Relative expression of ITGB1 and NOTCH1. A, B - Relative expression level of ITGB1 and NOTCH1 in the control, U87 oe-ITGB1 + AT13148, U87 $7^{\text {hh-ITGB1}}+$ AT13148, and U87 + AT13148 group. C, D - Relative protein expression level of ITGB1 and NOTCH1 in the control, U87 $7^{\text {oe-ITGB }}+$ AT13148, U87 $7^{\text {sh-ITGB1}}+$ AT13148, and U87+AT13148 group, ${ }^{*} p<0.05$ 
19 to $22 \mathrm{~g}$ (Figure $5 \mathrm{C}$ ). During the whole period of treatment, the tumor volume of mice in the AT13148 group was significantly lower than that in the control group at the $6^{\text {th }}, 9^{\text {th }}$, and $12^{\text {th }}$ day $(p<0.05)$ (Figure $5 \mathrm{D})$.

\section{AT13148 affects the expression of ITGB1 and NOTCH1 in tumor tissues}

We extracted the RNA and protein of tumor tissue to verify whether AT13148 can affect the expression of ITGB1 and NOTCH1 in vivo. The results of qRT-PCR indicated that, compared with that in the control group, the expression of ITGB1 and NOTCH1 genes in transplanted tumors decreased by $90 \%$ and $74 \%$, respectively $(p<0.05)$ (Figure $6 \mathrm{~A}$. The protein expressions of ITGB1 and NOTCH1 were also inhibited (Figure 6 B). Immunohistochemical results suggested that ITGB1

A

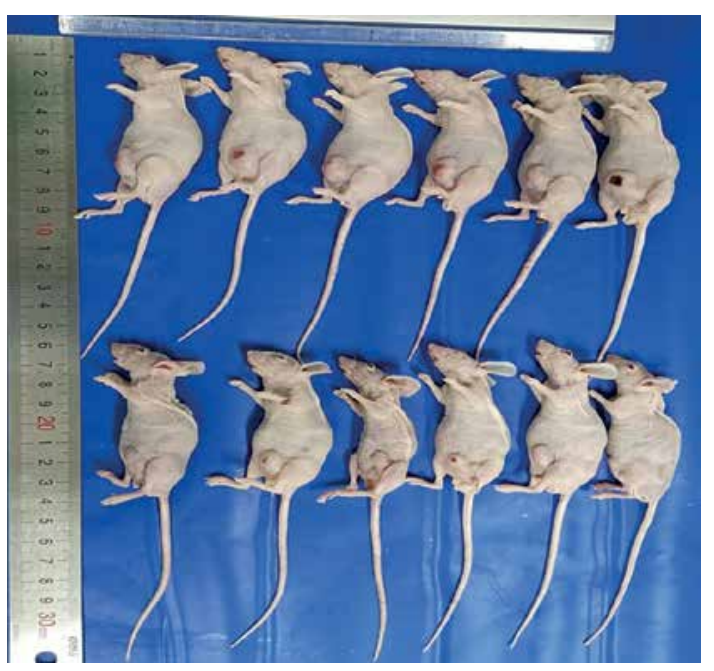

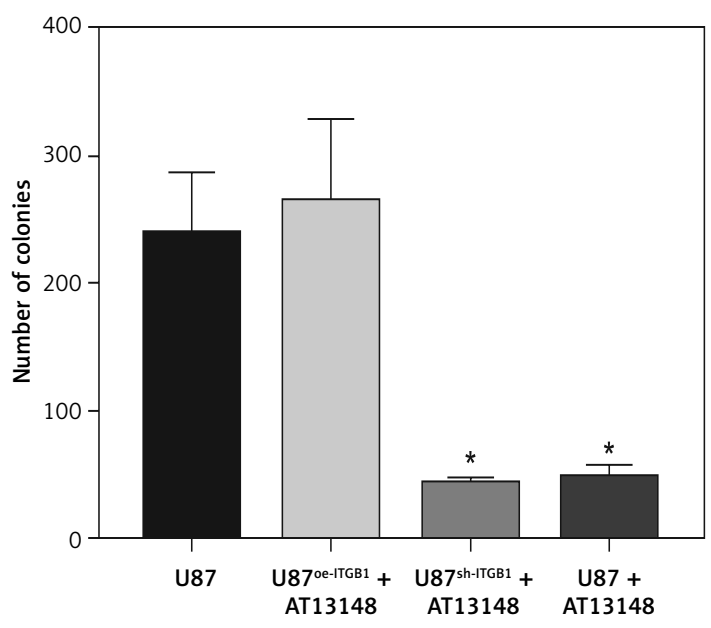

Figure 4. Number of colonies in the control, U870-ITGB1 + AT13148, U87 $7^{\text {sh-ITGB1 }}+$ AT13148, and U87 + AT13148 group, ${ }^{*} p<0.05$

B

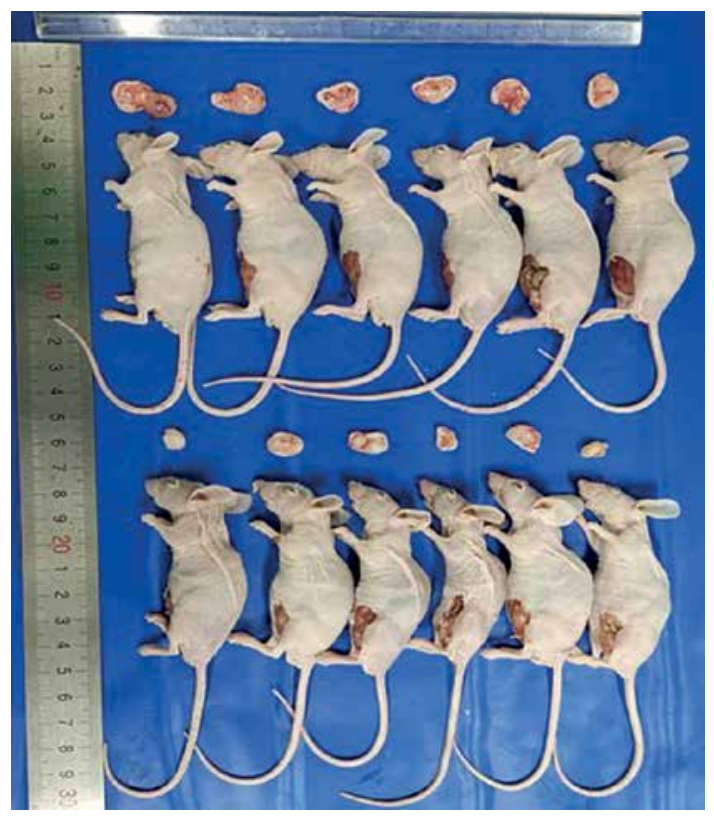

D

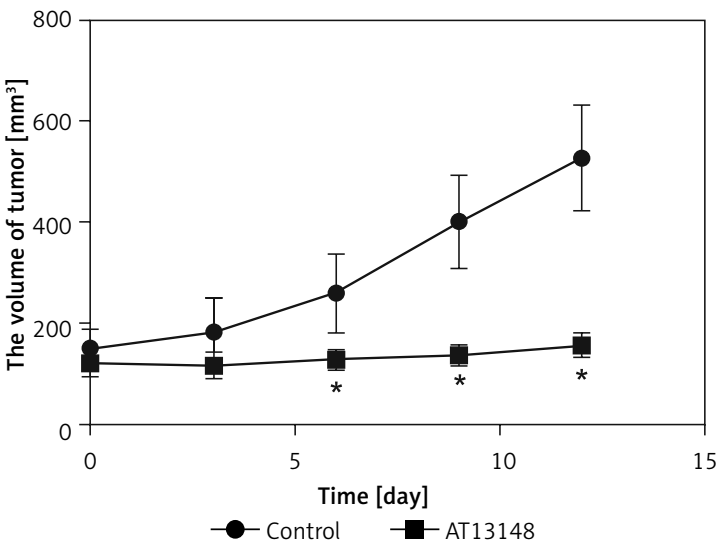

Figure 5. AT13148 affects the volume of tumors in nude mice. A, B - Nude mice with U87 xenograft tumor. The upper row consists of control group mice and the lower row consists of experimental group mice. C - Weight of mice. D - Tumor volume 

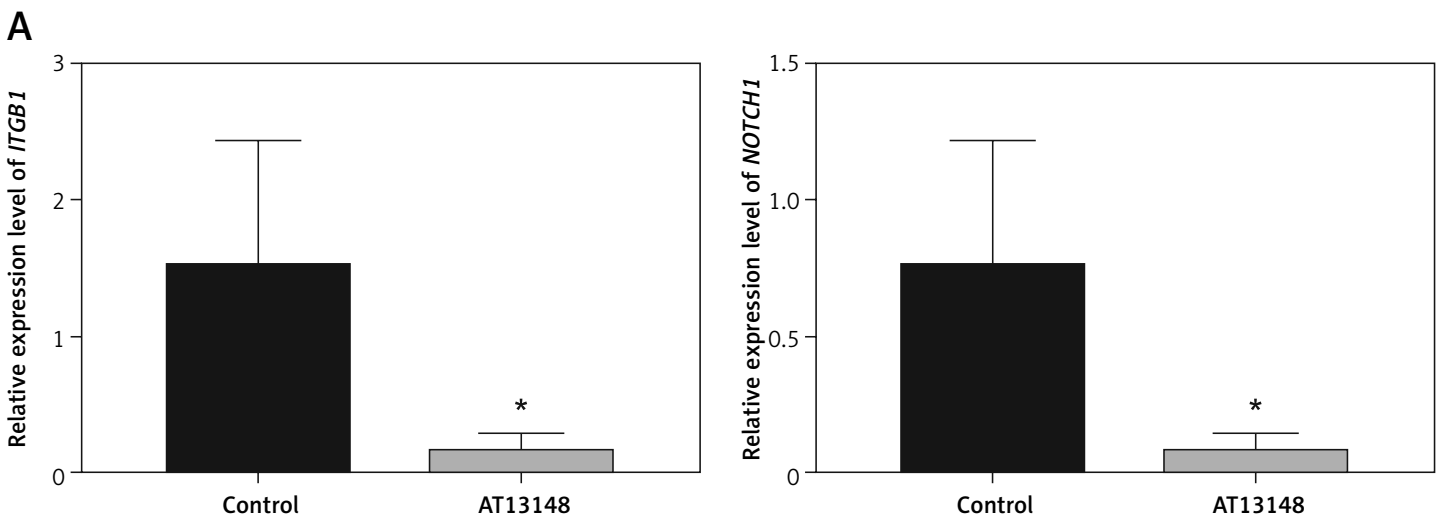

B

Control

AT13148

ITGB1

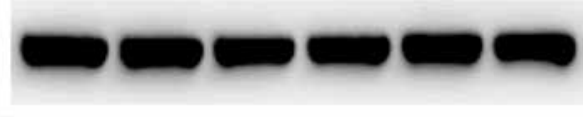

NOTCH1

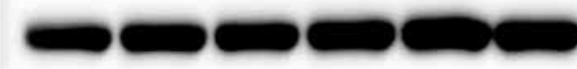

Actin

C
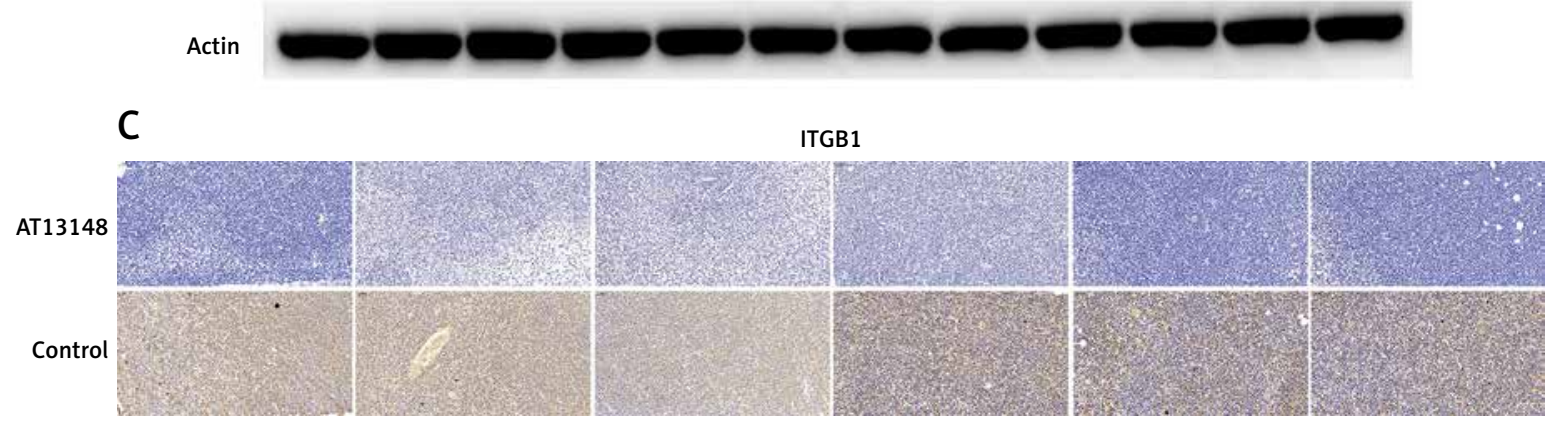

D

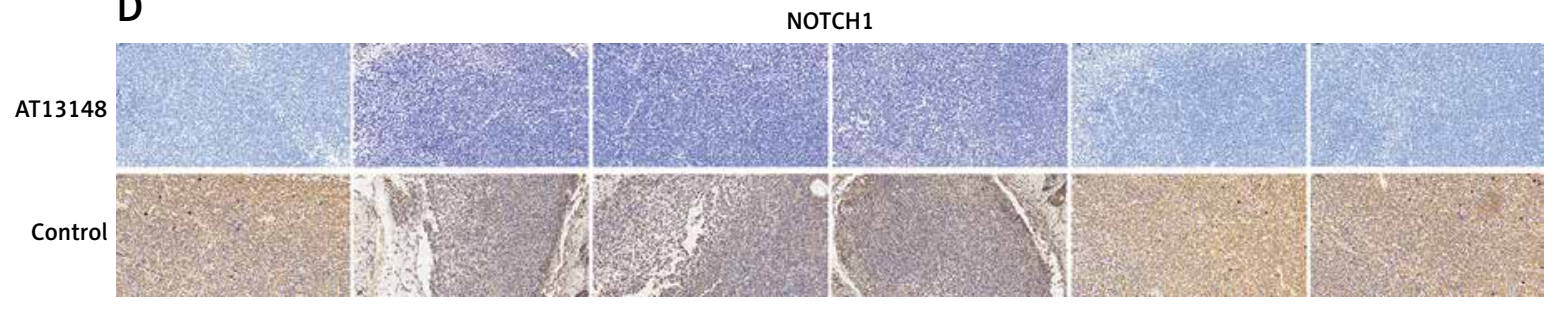

Figure 6. AT13148 regulates the expression of ITGB1 and NOTCH1 in the tumor. A - Relative expression levels of ITGB1 and NOTCH1 in different groups. B - Relative protein expression levels of ITGB1 and NOTCH1 in different groups. C, D - Immunohistochemical detection of ITGB1 and NOTCH1 in different groups, ${ }^{*} p<0.05$

and NOTCH1 were mainly expressed in the control group (Figures 6 C, D).

\section{Discussion}

Glioblastoma is a primary malignant glioma, which seriously threatens the life of patients. This study revealed that AT13148 could not only regulate the expression of ITGB1 and NOTCH1 but could also inhibit the growth of glioblastoma.

It has recently been shown that ITGB1 is involved in numerous processes including migration [27], cell proliferation [28], and invasion [27].
ITGB1 is involved in urokinase plasminogen activator-mediated angiogenesis through the urokinase plasminogen activator receptor (PLAUR) $[29,30]$. A study previously reported that ITGB1 can promote proliferation of glioma cells via feedback regulation of the Notch signaling pathway [31]. This study revealed that AT13148 could downregulate the expression of ITGB1 and inhibit AT13148, a multi-AGC kinase and ATP-competitive inhibitor. The AGC kinase family is important in cell proliferation and survival. AGC activity was significantly reduced in $\mathrm{HGC} 27$ tumors treated with AT13148. AT13148 as the target of AGC ki- 
nase showed good anti-gastric cancer activity in vitro and in vivo [32]. AGC kinase may play a similar role in this study. The study demonstrated that AT13148 has antiproliferative activity in a series of in vitro models harboring applicable genetic abnormalities, incorporating the gene of phosphate and tension homology deleted on chromosome ten $(P T E N)$, Kirsten rat sarcoma viral oncogene (KRAS), phosphatidylinositol-4,5-bisphosphate 3-kinase catalytic subunit alpha (PIK3CA) and human epidermal growth factor receptor 2 (HER2) aberrations. AT13148 caused substantial blockade of protein kinase $B$ (PKB), p70S6K, protein kinase $A$ (PKA), ROCK and SGK substrate phosphorylation. AT13148 also caused apoptosis in a time-dependent and concentration manner in tumor cells with clinically related genetic deficiency [33]. BT474 and PC3 cells treated with AT13148 affect the metabolites of gene proteins related to nitric oxide synthase (NOS) [34]. Activated Notch tumorigenesis was fueled by hampering the immune response or by NOS overexpression to mimic a pro-tumorigenic environment [35]. This suggests that AT13148 may affect the Notch signaling pathway by affecting NOS, which is also our future line of inquiry.

Due to the existence of the blood-brain barrier (BBB) and blood-brain tumor barrier (BBTB), most chemotherapeutic drugs can not directly reach the tumor site, which reduces the therapeutic effect of glioblastoma [36]. In this study, although the optimal concentration of AT13148 in U87 cells was determined according to the CCK- 8 assay, an in vivo experiment must be considered in the future. It is an important project to improve the permeability of AT13148 to BBB/BBTB for future in vivo research. Research has suggested that the active efflux of BBB/BBTB can be overcome by modifying the structure of candidate drugs and inhibiting the active efflux transport receptors. For instance, the small drug paclitaxel acquires increased BBB/ BBTB permeability by covalent conjugation of $\mathrm{N}$-docosahexaenoic acid [37]. Another perspective mainly includes a series of technologies to open or block the BBB/BBTB, such as focused ultrasound, radio-frequency microwaves, and laser interstitial thermotherapy [36].

In summary, the small molecule drug AT13148 regulates the expression of ITGB1 and NOTCH1 in the Notch pathway. In vivo experiments prove that it can affect the volume of tumors in nude mice. Our data suggested that AT13148 may serve as a potential anti-glioblastoma small molecule drug candidate in clinical medicine.

\section{Acknowledgments}

Yanan Li, Guosheng Han and Weijie Min contributed equally to this work.
This work was supported by the Natural Science Foundation of Shanghai (grant number 17ZR1438300).

\section{Conflict of interest}

The authors declare no conflict of interest.

\section{References}

1. Moots PL, Johnson MD, Jennings MT, Cmelak AT. Glioma and Other Neuroepithelial Neoplasms. John Wiley \& Sons, 2012.

2. Adamson DC, Rasheed BA, McLendon RE, Bigner DD. Central nervous system. Cancer Biomark 2010; 9: 193-210.

3. Martinez-Lage M, Sahm F. Practical implications of the updated WHO classification of brain tumors. Semin Neurol 2018; 38: 11-8.

4. Philbrick BD, Adamson DC. Early clinical trials of Toca 511 and Toca FC show a promising novel treatment for recurrent malignant glioma. Expert Opin Investig Drugs 2019; 28: 207-16.

5. Engelhardt M, Hansen C, Brendel B, et al. Real time neuronavigation using 3-D ultrasound and MRI in patients with brain tumor. In: Buzug TM, Holz D, Bongartz J, Kohl-Bareis M, Hartmann U, Weber S (eds.). Advances in Medical Engineering. Springer, Berlin, Heidelberg, 2007; 59-63.

6. Jeong H, Park J, Shim J-K, et al. Combined treatment with 2'-hydroxycinnamaldehyde and temozolomide suppresses glioblastoma tumorspheres by decreasing stemness and invasiveness. J Neurooncol 2019; 143: 69-77.

7. Batash R, Asna N, Schaffer P, Francis N, Schaffer M. Glioblastoma multiforme, diagnosis and treatment; recent literature review. Curr Med Chem 2017; 24: 3002-9.

8. Yuan L, Wang F, Zhang T, et al. Inhibition of glioma cell viability by jatrophone via NF-kB down-regulation and apoptosis inhibitor up-regulation. Arch Med Sci 2020; https://doi.org/10.5114/aoms.2020.94439.

9. Tykocki T, Eltayeb M. Ten-year survival in glioblastoma. A systematic review. J Clin Neurosci 2018; 54: 7-13.

10. Martina G. Targeted tumor therapy remixed - an update on the use of small-molecule drugs in combination therapies. Cancers 2018; 10: 155-75.

11. Yoko M, Dai S, Rie N, Atsushi Y, Ryutaro K. Immunotherapy for malignant tumors with focus on toll-like receptors. Oral Sci Int 2019; 16: 3-7.

12. Fu HG, Chen Y, Yu Q, Liu Y. A tumor-targeting Ru/polysaccharide/protein supramolecular assembly with high photodynamic therapy ability. Chem Commun (Camb) 2019; 55: 3148-51.

13. Ehebauer M, Hayward P, Martinez-Arias A. Notch signaling pathway. Sci STKE 2006; 2006: $\mathrm{cm} 7$.

14. Yao J, Fang X, Zhang C, et al. Astragaloside IV attenuates hypoxia-induced pulmonary vascular remodeling via the Notch signaling pathway. Mol Med Rep 2021; 23: 89.

15. Zamurovic N, Cappellen D, Rohner D, Susa M. Coordinated activation of notch, Wnt, and transforming growth factor-beta signaling pathways in bone morphogenic protein 2-induced osteogenesis. Notch target gene Hey1 inhibits mineralization and Runx2 transcriptional activity. J Biol Chem 2004; 279: 37704-15.

16. Porcelli L, Mazzotta A, Garofoli M, et al. Active notch protects MAPK activated melanoma cell lines from MEK 
inhibitor cobimetinib. Biomed Pharmacother 2021; 133: 111006.

17. Liu Z, Ma T, Duan J, Liu X, Liu L. MicroRNA-223-induced inhibition of the FBXW7 gene affects the proliferation and apoptosis of colorectal cancer cells via the Notch and Akt/mTOR pathways. Mol Med Rep 2021; 23: 154-63.

18. Cuervo H, Nielsen CM, Simonetto DA, Ferrell L, Rong AW. Endothelial Notch signaling is essential to prevent hepatic vascular malformations in mice. Hepatology 2016; 64: 1302-16.

19. Qiang L, Wu T, Zhang HW, Lu N, Guo Q-L. HIF-1 is critical for hypoxia-mediated maintenance of glioblastoma stem cells by activating Notch signaling pathway. Cell Death Differ 2012; 19: 284-94.

20. Saito N, Aoki K, Hirai N, et al. Effect of Notch expression in glioma stem cells on therapeutic response to chemo-radiotherapy in recurrent glioblastoma. Brain Tumor Pathol 2015; 32: 176-83.

21. Min W, Li Y, Zhang Y, et al. Role of the anti-glioma drug AT13148 in the inhibition of Notch signaling pathway. Gene 2015; 573: 153-9.

22. Shang $M, X u X$, Zhang $M$, Yang H. Long non-coding RNA linc-ITGB1 promotes cell proliferation and migration in human hepatocellular carcinoma cells. Exp Ther Med 2017; 14: 4687-92.

23. Yang J, Hou Y, Zhou M, et al. Twist induces epithelial-mesenchymal transition and cell motility in breast cancer via ITGB1-FAK/ILK signaling axis and its associated downstream network. Int J Biochem Cell Biol 2016; 71: 62-71.

24. Yap TA, Walton MI, Grimshaw KM, et al. AT13148 is a novel, oral multi-AGC kinase inhibitor with potent pharmacodynamic and antitumor activity. Clin Cancer Res 2012; 18: 3912-23.

25. Wang P, Wang Y, Hang B, Zou X, Mao JH. A novel gene expression-based prognostic scoring system to predict survival in gastric cancer. Oncotarget 2016; 7 55343-51.

26. Qiao Z, Cheng SX, Zhang XZ, Zhuo RX. Water soluble polymer protected lipofectamine 2000/DNA complexes for solid-phase transfection. Macromol Biosci 2009; 9 : 1262-71.

27. Wang N, Chang LL. Maspin suppresses cell invasion and migration in gastric cancer through inhibiting EMT and angiogenesis via ITGB1/FAK pathway. Hum Cell 2020; 33: 663-75.

28. Liu W, Zhang GQ, Zhu DY, et al. Long noncoding RNA ZFPM2-AS1 regulates ITGB1 by miR-1226-3p to promote cell proliferation and invasion in hepatocellular carcinoma. Eur Rev Med Pharmacol Sci 2020; 24: 7612-20.

29. Malin H, Anita K, Mikael I, et al. Keratinocytes and head and neck squamous cell carcinoma cells regulate urokinase-type plasminogen activator and plasminogen activator inhibitor-1 in fibroblasts. Anticancer Res 2013; 33: 3113-8.

30. Bettina G, Sumito S, Charlotte K, et al. Overexpression of the urokinase receptor splice variant uPAR-del $4 / 5$ in breast cancer cells affects cell adhesion and invasion in a dose-dependent manner and modulates transcription of tumor-associated genes. Biol Chem 2012; 393: 1449-55.

31. Min W, Zou C, Dai D, et al. Integrin beta 1 promotes glioma cell proliferation by negatively regulating the notch pathway. I Oncol 2020; 2020: 8297017.

32. Xi Y, Niu J, Shen Y, Li D, Peng X, Wu X. AT13148, a first-inclass multi-AGC kinase inhibitor, potently inhibits gas- tric cancer cells both in vitro and in vivo. Biochem Biophys Res Commun 2016; 478: 330-6.

33. Yap TA, Walton MI, Grimshaw KM, et al. The novel clinical candidate AT13148 is an oral multi-AGC kinase inhibitor with potent pharmacodynamic and antitumor activity and demonstrates a mechanism of action distinct from AKT inhibitors. Cancer Res 2012; 72: abstract 928.

34. Pal A, Asad Y, Ruddle R, et al. Metabolomic changes of the multi (-AGC-) kinase inhibitor AT13148 in cells, mice and patients are associated with NOS regulation. Metabolomics 2020; 16: 50.

35. Villegas SN, Gombos R, García-López L, et al. PI3K/Akt cooperates with oncogenic Notch by inducing nitric oxide-dependent inflammation. Cell Rep 2018; 22: 2541-9.

36. Wang D, Wang C, Wang L, Chen Y. A comprehensive review in improving delivery of small-molecule chemotherapeutic agents overcoming the blood-brain/brain tumor barriers for glioblastoma treatment. Drug Deliv 2019; 26: 551-65.

37. Bradley MO, Webb NL, Anthony FH, et al. Tumor targeting by covalent conjugation of a natural fatty acid to paclitaxel. Clin Cancer Res 2001; 7: 3229-38. 